anthropology \& materialism

\section{Anthropology \& Materialism}

A Journal of Social Research

$3 \mid 2016$

Utopia: The Elsewhere and The Otherwise

\title{
Entre autorité et émancipation
}

L'utopie pédagogique de Gramsci dans le $12^{\mathrm{e}}$ Cahier de prison

Between Authority and Emancipation: The Utopian Pedagogy of Gramsci in the 12th Prison Notebook

Entre autoridad y emancipación. La pedagogía utópica de Gramsci en el 12 cuaderno de prisión

\section{Stéphanie Roza}

\section{(2) OpenEdition \\ Journals}

\section{Édition électronique}

URL : https://journals.openedition.org/am/622

DOI : 10.4000/am.622

ISSN : 2364-0480

\section{Éditeur :}

CETCOPRA, CRASSH - Center for Research in the Arts Social Sciences and Humanities, Fakultät

Gestaltung - Universität der Künste Berlin

Référence électronique

Stéphanie Roza, "Entre autorité et émancipation », Anthropology \& Materialism [En ligne], 3 | 2016, mis en ligne le 12 novembre 2016, consulté le 21 septembre 2021. URL : http://journals.openedition.org/am/ 622 ; DOI : https://doi.org/10.4000/am.622

Ce document a été généré automatiquement le 21 septembre 2021.

Tous droits réservés 


\title{
Entre autorité et émancipation
}

\author{
L'utopie pédagogique de Gramsci dans le $12^{\mathrm{e}}$ Cahier de prison \\ Between Authority and Emancipation: The Utopian Pedagogy of Gramsci in the \\ 12th Prison Notebook \\ Entre autoridad y emancipación. La pedagogía utópica de Gramsci en el 12 \\ cuaderno de prisión
}

Stéphanie Roza

1 Antonio Gramsci ne s'est jamais revendiqué de l'utopie. Dans les Cahiers de prison, il n'utilise le terme que dans le sens courant et péjoratif de chimère, ou de désir irréalisable. En revanche, la question pédagogique est une question centrale chez lui ${ }^{1}$. Au-delà du cadre de l'école proprement dite, elle est intégrée dans le thème bien plus large de la lutte pour l'hégémonie, c'est-à-dire, pour le dire très brièvement, de la conquête par une classe de ce pouvoir symbolique mais indispensable qu'est la domination culturelle. Seule une telle domination peut produire le consentement des individus à l'ordre social porté par ladite classe. Gramsci l'exprime explicitement dans le Cahier 10 : «(..) le rapport pédagogique ne peut être limité aux rapports spécifiquement "scolaires" (...) Tout rapport d'hégémonie est nécessairement un rapport pédagogique (...) »². Ainsi, pour Gramsci, le rapport pédagogique n'est pas à l'œuvre seulement à l'école. Syndicats, partis politiques, églises, journaux, etc. sont autant de lieux où il opère. C'est pourquoi la conquête de l'hégémonie culturelle doit être à ses yeux l'objectif premier de son parti, le Parti communiste italien représentant les intérêts des classes dominées. Le Cahier 12, vraisemblablement écrit entre la mi-1932 et la mi-1933, est presque entièrement consacré à cette question. Il trace les contours d'une école plus conforme aux vœux du penseur socialiste, constituant par là même le terrain d'application virtuel d'application de ses principes pédagogiques.

Or il semble comme on va s'efforcer de le montrer, que ce texte présente plusieurs aspects d'une utopie éducative. Écrit sous le fascisme, à un moment où Gramsci est, de fait, coupé de toute possibilité d'intervention directe sur le monde réel, il campe une institution imaginaire, faisant l'économie d'une réflexion sur les moyens qui pourraient permettre de passer de l'école réelle à l'école voulue. Cette absence de médiation entre le 
présent rejeté et l'avenir désiré, cette rupture entre l'auteur et son temps, met le lecteur sur la piste de l'utopie.

On se propose donc une relecture du Cahier 12 au prisme de ses aspects utopiques, qui permettra de juger dans quelle mesure son auteur affronte les mêmes ambiguïtés que d'autres pédagogues en Utopie, et de s'interroger sur la manière dont il tente de les surmonter.

\section{Gramsci et la théorie de l'école unitaire}

4 A première vue, le Cahier 12 contient un projet d'institution d'une " école unique " pour tous les enfants d'une même génération, projet que l'on est tenté de lire comme une alternative à la réforme Gentile (nom du ministre fasciste de l'éducation, par ailleurs philosophe) de 1923. Cette réforme, rappelons-le, avait pour objectif de réaffirmer le caractère hiérarchisé, centralisé et élitiste de l'école italienne, centrée sur la formation de la classe dirigeante du pays. Affirmés avec force dans le décret-loi Casati du 13 novembre 1859, ces traits avaient été atténués par des réformes ultérieures : la réforme Gentile fut donc, de l'aveu même de son auteur, pensée dans un esprit de "restauration ». La nomination de tous les fonctionnaires devait désormais relever de la tutelle suprême du ministre de l'instruction publique, et les professeurs, être placés sous la surveillance étroite de leurs supérieurs hiérarchiques. L'ensemble des fonctionnaires du ministère de l'instruction publique devenait ainsi une courroie de transmission idéologique pour le pouvoir fasciste. Une dure sélection était également érigée en principe, et la majeure partie des petits Italiens était censée quitter l'école à neuf ans, à la fin du cycle préparatoire. Les pédagogues devaient miser avant tout sur la spontanéité des enfants et leur affectivité ; l'accent était mis sur l'exaltation du patriotisme à travers l'enseignement d'un certain roman national et la valorisation des traditions italiennes, au détriment de l'apprentissage du raisonnement et des connaissances scientifiques. L'enseignement de la doctrine chrétienne occupait également une place de choix. La philosophie interviendrait plus tard, à un moment où les enfants du peuple auraient déjà quitté l'école. L'étanchéité des enseignements de l'école primaire et des études secondaires et supérieures était consciemment mise en œuvre. Pour les enfants des milieux populaires les plus méritants, Gentile créait une "école complémentaire » qui durait trois ans et dont le diplôme ne permettait pas d'accéder à un niveau supérieur d'études. Le lycée, réservé aux enfants de "l'élite ", dispenserait, lui, une culture humaniste nécessaire à la formation des classes dirigeantes: grec, latin, littérature italienne. Un autre aspect important de la réforme résidait dans la formation des maîtres eux-mêmes: le ministre voulait leur donner un important bagage philosophique, et les mettre en capacité d'assumer un enseignement pluridisciplinaire. Leur formation, payante, était extrêmement exigeante à tous égards. Enfin, par la création du lycée féminin, étaient entérinés la séparation des sexes et le cantonnement des femmes à leur rôle domestique ${ }^{3}$.

5 Le Cahier 12 est-il donc un modèle concurrent de celui de Gentile? En partie, oui, sans doute. Mais à y regarder de plus près, les choses ne sont pas si simples. Tout d'abord, il est remarquable - bien qu'il n'ait pas été souvent remarqué - que ce supposé projet de réforme n'est jamais vraiment présenté comme tel. Gramsci l'introduit en ces termes ambigus : « La crise [de l'école] trouvera une solution qui devrait, rationnellement, suivre la ligne que voici (...) $»^{4}$. La résolution des problèmes semble donc renvoyée à un avenir indéterminé : en ce sens, le passage pourrait être considéré comme une prévision 
lointaine ou une orientation générale. Toutefois, le luxe des détails et la précision du plan rendent une telle lecture peu satisfaisante.

6 Tout porte plutôt à croire que l'on a affaire ici à une expérience de pensée, à un pur exercice de la raison politique appliqué à la question éducative, ce qui a de quoi étonner de la part d'un chef de parti comme Gramsci. Pourtant, plusieurs éléments viennent renforcer une telle hypothèse : tout d'abord, la situation de l'auteur, député emprisonné par le régime mussolinien et par là réduit à l'impuissance politique ; ensuite, la nature du texte, recueil de réflexions dont Gramsci ne pouvait certainement pas prévoir le destin, qu'il rédigeait probablement pour lui-même plutôt que comme brouillon d'un projet de loi ; enfin, l'audace des propositions, sur laquelle on va revenir, qui contraste de façon criante avec le contexte politique dans lequel celles-ci sont énoncées, dans lequel plus rien ne semble possible.

7 L'idée d'une expérience de pensée sous la plume d'un homme d'action paraîtra peut-être moins surprenante si l'on rappelle certains précédents. Par ces caractéristiques, le Cahier 12 rappelle en effet un autre texte lui aussi resté à l'état de brouillon, écrit par un révolutionnaire qui se trouvait, pour des raisons différentes, dans un désarroi politique analogue à celui du dirigeant italien. Il s'agit des Institutions républicaines de Saint-Just, rédigées en pleine Terreur, au printemps 1794, alors que selon les termes mêmes de leur auteur, "la Révolution est glacée » et la chute est proche. Ces institutions d'une République égalitaire et réconciliée avec elle-même, bien éloignée des cruelles nécessités de la Terreur, étonnent par leur caractère idyllique. Elles présentent d'évidents aspects utopiques. Miguel Abensour a pu expliquer la démarche de leur auteur en suggérant que " "L'utopie", au sens d'une expression des limites de la conscience possible dans une société donnée, se situe au point de chute d'une action politique qui se dévoile soudainement à son auteur comme impossible $»^{5}$. Les Institutions républicaines seraient ainsi l'expression des espérances politiques de Saint-Just à un moment où leur réalisation lui semblait, à juste titre, plus lointaine que jamais. L'appréciation pourrait valoir pour le Cahier 12 : la communauté (relative) de situation de Gramsci, prisonnier politique, et de Saint-Just, prisonnier de la logique révolutionnaire et de la «force des choses", aurait conduit ces deux dirigeants avides de changement social radical à voir de façon similaire une issue dans la prospective utopique.

8 Cette supposition se confirme si on entre dans les détails du projet lui-même. Rappelons ses grands traits en quelques mots : Gramsci s'oppose à la réforme en cours, qui entend se débarrasser de "toute espèce d'école désintéressée ", ou plutôt qui cherche à "n'en maintenir qu'un modèle réduit réservé à une petite élite d'hommes et de femmes privilégiés $[. ..] »^{6}$. Il plaide au contraire pour une école unique «de culture générale, humaniste et formatrice, alliant justement le développement des capacités manuelles (techniques, industrielles) et celui des capacités de travail intellectuel ${ }^{7}$. La totalité des enfants d'une génération serait prise en charge très tôt, à partir de la maternelle, et jusqu'à 15 ou 16 ans, dans un cadre où tous recevraient strictement la même formation. Cette dernière combinerait instruction et éducation, dans la mesure où, en plus des " premières notions instrumentales - lecture, écriture, calcul, géographie, histoire ", elle serait porteuse d'une morale civique afin que les jeunes soient très tôt au fait de leurs "droits et devoirs »". Il s'agit donc d'édifier les jeunes gens dans un cadre généraliste et ambitieux, repoussant la professionnalisation à plus tard. En effet, ce n'est qu'à l'issue de ces années de formation commune que chacun pourrait se spécialiser en fonction de ses compétences et de ses désirs dans une visée qui pourrait alors, selon les individus, être 
purement intellectuelle, ou au contraire manuelle et technique. Au lieu, avec Gentile, de pérenniser en la légitimant l'inégalité culturelle et sociale, le projet tend donc à la réduire autant qu'il est possible de le faire dans le cadre de l'instruction publique.

9 D'après ces caractéristiques, les réflexions de Gramsci semblent annoncer, comme plusieurs commentateurs l'ont souligné, le plan Langevin-Wallon de la Libération, en France. On y retrouve en effet deux aspects essentiels : d'une part, la dimension civique de l'éducation nationale, qui s'oppose à la volonté fasciste de tirer de la religion chrétienne et d'un nationalisme exacerbé les fondements de la morale des futurs adultes. Les auteurs du plan de 1947 affirment sans ambiguïté : « [la] formation civique de la jeunesse est l'un des devoirs fondamentaux d'un Etat démocratique et c'est à l'enseignement public qu'il appartient de remplir ce devoir $»^{9}$. Comme chez Gramsci, c'est la conscience d'appartenir à une communauté politique, induite par l'éducation nationale, qui doit cimenter le lien social. D’autre part, inséparable de ce premier principe, la visée égalitaire, qui passe par l'accès de tous au savoir «désintéressé» est affirmée avec la même force: "L'enseignement doit offrir à tous d'égales possibilités de développement, ouvrir à tous l'accès à la culture, se démocratiser moins par une sélection qui éloigne du peuple les plus doués que par une élévation continue du niveau culturel de l'ensemble de la nation $»^{10}$.

10 Quant à l'idée de "l'école unique», elle fait penser, dans sa forme comme dans ses ambitions, à la réforme Haby ayant instauré le collège unique en 1975. Sur la base d'un même constat de "crise » de l'éducation, le ministre français proposera lui aussi d'y mettre un terme en abolissant la différence entre l'école du pauvre, professionnalisante, et l'école de l'élite, en instaurant un creuset unique de formation. Pour cela, la formation secondaire reposera "sur un équilibre des disciplines intellectuelles, artistiques, manuelles, physiques et sportives» devant permettre «de révéler les aptitudes et les goûts $»^{11}$, comme dans le Cahier 12.

11 Précédant de quelques décennies ces réformes importantes, les suggestions de Gramsci seraient, dans cette perspective, tout au plus, de judicieuses anticipations. Elles n'auraient à la rigueur d'utopique que les conséquences parfaitement égalitaires qu'elles prévoyaient, suite à la mise en pratique d'un tel plan.

12 Cependant, point décisif, Gramsci précise que la prise en charge de la jeunesse ne doit pas se limiter aux heures de cours: «ce type d'école devrait être une école-collège, comprenant dortoirs, réfectoires, bibliothèques spécialisées, salles prévues pour un travail de séminaire, etc. $»^{12}$. Ce dernier aspect permet au lecteur contemporain de prendre la mesure de la charge utopique du projet gramscien. Par lui, on comprend en effet que l'objectif dépasse celui des écoles publiques des pays occidentaux modernes. L'auteur ne se contente pas d'imaginer des internats pour certains enfants qui en auraient besoin, mais d'en étendre la fréquentation à l'ensemble des membres d'une génération. L'ambition est donc celle d'une formation "totale», dans tous les domaines et à tous les moments de la vie: il s'agit d'assumer "toute entière" la fonction « d'éducation et de formation des jeunes générations, car c'est seulement ainsi qu'elle peut englober toutes les générations sans divisions de groupes ou de castes ${ }^{13}$.

13 Gramsci cherche ainsi à éviter, en des termes dont on a pu aussi souligner la proximité avec ceux de Bourdieu ${ }^{14}$, que les enfants des classes populaires ne soient pénalisés par la pauvreté du capital culturel familial. Il souligne l'apport intellectuel que les enfants des milieux privilégiés absorbent " comme on dit, avec l'air qu'ils respirent $»^{15}$, dans le milieu familial, quand les enfants des classes populaires, en particulier dans les campagnes, en sont privés. Pourtant, sans doute davantage que Bourdieu ou Paul Langevin, cette volonté 
de prise en charge globale rappelle, là encore, l'ambition révolutionnaire de créer, par l'éducation, un citoyen nouveau libéré de toutes les entraves de l'ancien ordre des choses. Elle évoque en particulier le projet de Lepelletier de Saint-Fargeau, lu à la barre de la Convention par Robespierre le 13 juillet 1793 après l'assassinat de son auteur. Le projet, qui visait ouvertement à " créer un nouveau peuple $»^{16}$ ne fut jamais appliqué, notamment parce qu'il aurait coûté trop cher. Il prévoyait cependant, comme celui de Gramsci, l'éducation commune, hors des familles, à tous les enfants (de 5 à 11 ans pour les filles et de 5 à 12 ans pour les garçons) financée par un impôt progressif, et obligatoire sous peine pour les parents de perdre leurs droits civiques. Les enfants devaient tous être logés et éduqués ensemble pendant tout cet intervalle. Sans pouvoir rentrer dans les détails de ce plan comprenant à la fois l'éducation physique, théorique et morale de la jeunesse, rappelons seulement son but affiché : « Ainsi, depuis cinq ans jusqu'à douze, c'est-à-dire dans cette portion de la vie si décisive pour donner à l'être physique et moral la modification, l'impression, l'habitude qu'il conservera toujours, tout ce qui doit composer la République, sera jeté dans un moule républicain ${ }^{17}$.

Pour Lepelletier, il s'agissait donc bien de fabriquer, presque littéralement, des individus destinés à servir la République, et ce, en dépit de leurs parents comme le montre ce commentaire : «[...] je remarque avec peine que jusqu'à six ans l'enfant échappe à la vigilance du législateur, et que cette portion importante de la vie reste abandonnée aux préjugés subsistants et à la merci des vieilles erreurs ${ }^{18}$. Cette dernière préoccupation, et cette méfiance recoupent parfaitement celles de Gramsci, qui affirme à son tour que les connaissances et les valeurs dispensées par l'école unitaire "constituent les éléments primordiaux d'une nouvelle conception du monde qui entre en lutte contre les conceptions véhiculées par les différents milieux sociaux traditionnels, autrement dit contre les conceptions qu'on peut qualifier de folkloriques $»^{19}$. Par là, l'auteur pointe en même temps les travers de l'éducation fasciste et son exaltation des valeurs traditionnelles, de « l'âme » italienne.

Assumant la prise en charge complète de la jeunesse, visant l'édification morale du futur citoyen en même temps qu'un développement optimal de ses capacités intellectuelles et physiques, dans un cadre d'où toute différenciation sociale aurait été exclue, le plan d'éducation gramscien n'était pas un plan applicable dans l'ordre existant au moment de son élaboration. Il n'était peut-être pas non plus applicable dans l'ordre social qui précéda, ni dans celui qui a suivi. Le bilan en demi-teinte des réformes de l'école française de la deuxième moitié du XXe siècle ne contredit certes pas l'impression selon laquelle l'objectif de l'égalisation absolue des conditions par l'éducation est demeuré une utopie, au sens péjoratif cette fois, dans une société qui produit et reproduit l'inégalité à chaque génération. Dans cette perspective, le caractère hybride des changements imaginés, inspirés tantôt par l'éducation du passé qui contraint et même formate, tantôt par les pédagogies actives, plus libérales, qui feront florès après la mort de Gramsci, renforce le caractère atemporel, insituable, du plan dans sa globalité. Inassignable, d'aucun temps ni d'aucun lieu, « l'école unitaire » a bien les attributs d'une utopie pédagogique.

\section{Utopie(s) pédagogique(s)}

Le Cahier 12 semble donc un lointain héritier de ce que François Hincker a appelé « l'effet d'utopie de la Révolution française $»^{20}$. Le discours gramscien est imprégné de la même intuition utopique fondamentale que ceux des révolutionnaires des années 1789-1794: 
l'idée selon laquelle les institutions publiques peuvent et doivent gommer les inégalités sociales en même temps qu'elles uniront les futurs citoyens dans un commun amour du bien public, et un commun dévouement à la collectivité. Cette proximité avec l'utopie révolutionnaire permet de jeter un pont entre le théoricien sarde et la tradition utopique en général, y compris dans sa période pré-révolutionnaire. On peut montrer en effet que le contenu même des propositions du Cahier 12 comporte plusieurs aspects qui permettent de mettre en évidence, sinon une filiation doctrinale, du moins une communauté de vue assez forte entre ces différentes générations de penseurs de la transformation sociale.

Le premier de ces aspects est la critique de l'école existante. Gramsci rejette le rapport purement utilitaire à l'éducation qui conduit à réserver «l'orientation humaniste de la culture générale fondée sur la tradition gréco-romaine $»^{21}$ aux enfants de l'élite sociale, tandis que l'écrasante majorité des enfants devrait se contenter d'écoles "professionnelles spécialisées » qui orientent chacun trop tôt vers sa future profession. Cette critique, ainsi que l'alternative qui lui est proposée, à savoir une formation non spécialisée, à la fois intellectuelle et manuelle, théorique et pratique, pour tous, ne rappelle pas seulement la tradition républicaine radicale à la française. Elle ressemble aussi beaucoup à la formation des citoyens de l'utopie. Chez More, en effet, tous les enfants, filles et garçons, sont initiés aux travaux des champs en même temps qu'ils reçoivent leur éducation théorique; dans La Cité du Soleil de Campanella également, une éducation de l'esprit et du corps est dispensée de façon identique aux enfants, la spécialisation n'intervenant que dans un deuxième temps. Dans beaucoup d'autres utopies communautaires, le même principe est affiché. Cabet écrit ainsi: "Tous les Icariens, sans distinction de sexes et de professions, reçoivent la même éducation générale ou élémentaire, qui embrasse les éléments de toutes les connaissances humaines $»^{22}$.

De même, l'importante dimension morale et civique de la formation selon Gramsci, évoquée plus haut, s'oppose à la conception instrumentale de l'école élémentaire fasciste qui ne sert qu'à inculquer des compétences techniques. Il ne s'agit pas seulement de produire les futurs rouages de la machine productive, mais d'éduquer les membres d'une collectivité sociale soudée par un ensemble de buts et de valeurs communs. Cet objectif aussi recoupe les préoccupations des utopistes: More le premier, encore une fois, confie la « première instruction » des enfants et des adolescents à des prêtres élus par le peuple, instruction qui « porte sur le caractère et la morale autant que sur les lettres ${ }^{23}$.

19 Chez Gramsci comme dans L'Utopie, l'égalité de la formation primitive est bien sûr le prélude à une spécialisation qui marque la fin de l'indifférenciation, et l'émergence d'une élite intellectuelle déchargée des tâches productives. Les études universitaires pour Gramsci restent réservées aux plus brillants, comme dans le récit de Raphaël Hythlodée où une minorité d'Utopiens sont choisis pour se consacrer à l'étude, et sont dispensés du service agricole. Mais cette différenciation par le mérite n'interdit pas aux citoyens du commun de continuer à apprendre, bien au contraire. L'abolition de la coupure existentielle entre le temps de la jeunesse, temps de la formation, et l'âge adulte, temps de la productivité, est une idée commune à Gramsci et à de nombreux utopistes. L'un des aspects frappants du plan du Cahier 12 est en effet l'institution des "académies " réservées aux adultes. Ce sont des lieux qui doivent permettre la rencontre « entre les intellectuels et le peuple", des lieux de collaboration et d'échange où les individus productifs doivent pouvoir trouver les moyens de parfaire leurs connaissances et participer à la recherche et au travail scientifique. Gramsci prend la question très au 
sérieux puisqu'il imagine un maillage académique serré du territoire, depuis les « cercles locaux urbains et ruraux » jusqu'aux « centres nationaux » en passant par les « sections régionales $»^{24}$.

Sa réflexion, là encore, se situe dans le droit fil des descriptions utopiques: More avant lui imagine que tous les Utopiens sont cultivés, et qu'ils se pressent tous les matins aux multiples conférences qui ont lieu avant le lever du soleil ; Campanella, lui, recouvre 6 des 7 enceintes de la Cité du Soleil de peintures figurant l'ensemble des connaissances scientifiques accumulées par l'intelligence humaine, afin que chacun puisse à tout moment les apprendre ou en retrouver la mémoire. A la fin du XIXe siècle encore, Theodor Hertzka dans son Freiland estime que le système d'instruction doit permettre que «l'ouvrier le plus simple possède non seulement son métier, depuis le maniement des machines jusqu'à la connaissance des sources de rapports et des débouchés, mais dispose encore, dans une mesure importante, d'une instruction générale $»^{25}$.

21 Le résultat espéré par Gramsci livre la clé de cette proximité : comme il l'écrira à sa femme, l'idéal de «l'homme nouveau » visé par ses efforts n'est qu'une version actualisée de l'humaniste de la Renaissance, c'est-à-dire le type incarné, dans une large mesure, par des hommes comme Thomas More, érudit et ami d'Erasme, ou Tomaso Campanella, égyptologue, botaniste, zoologue, géographe et philosophe. Gramsci écrit: «L'homme moderne devrait être une synthèse de traits que l'on a hypostasié en caractères nationaux: l'ingénieur américain, le philosophe allemand, le politique français, en recréant, pour ainsi dire, l'homme italien de la Renaissance, le type moderne de Léonard de Vinci " ${ }^{26}$. Etonnante nostalgie d'un prestigieux passé européen chez ce marxiste revendiqué.

\section{Contrainte et liberté}

La communauté de vues ainsi reconstituée ne peut cependant pas masquer des divergences assez importantes sur le terrain du dilemme entre autorité et émancipation. Elles sont le lieu de tentatives d'ajustement parfois difficiles entre les fins et les moyens.

A première vue, les utopies paraissent se faire les avocates de la pédagogie sans effort et de l'apprentissage en s'amusant. Comme le résume Anne-Marie Drouin-Hans : «Facilité, rapidité, efficacité, telle est la triade sur laquelle repose la pédagogie des utopies ${ }^{27}$. Même si aucune méthode pédagogique originale n'est vraiment décelable dans ces textes, les citoyens d'Utopie sont globalement décrits comme apprenant dans le plaisir et le jeu. Rien ne leur semble à charge, pas même le lever quotidien à quatre heures du matin pour aller écouter de longues conférences. Au contraire, Gramsci expose l'irréductible dimension contraignante et même douloureuse de l'apprentissage, notamment pour les enfants des milieux populaires. Ce penseur sarde d'origine paysanne parle sans aucun doute en connaissance de cause. Mais on peut voir aussi dans cette prise de position une réaction à l'enthousiasme de Gentile pour la nature immédiate de l'enfant et le recours à ses affects pour l'instruire. Comme le travail manuel, les études, affirme au contraire Gramsci, " sont elles aussi un métier, et très pénible, non seulement intellectuel, mais aussi musculaire et nerveux; c'est un procès d'adaptation, c'est une disposition qu'on acquiert grâce à l'effort, l'ennui et même la souffrance $»^{28}$. Il convient avant tout d'en convaincre les familles où les métiers manuels prévalent, pour qu'elles comprennent les efforts exigés de leurs enfants. La discipline, la coercition même sont des leviers indispensables pour le pédagogue dans la mesure où « on a affaire à de jeunes enfants, auxquels il est nécessaire d'inculquer certaines habitudes de soin, d'exactitude, de 
correction même physique, de concentration psychique sur des sujets déterminés, qu'on ne peut acquérir sans une répétition mécanique d'actes disciplinés et méthodiques ${ }^{29}$. Il est difficile d'être plus clair.

Dans le même ordre d'idées, Gramsci s'oppose, au moins partiellement, aux pédagogies utopiques sur la question des contenus à transmettre. Un certain nombre d'utopistes de la période pré-révolutionnaire s'accordent plus ou moins clairement à rejeter l'éducation scolastique dans laquelle ils ont souvent été instruits, et notamment un de ses pivots, l'apprentissage des langues anciennes, le latin et le grec. More en particulier, comme le souligne Anne-Marie Drouin-Hans, tourne en dérision « les subtilités de la logique et les abstractions de la scolastique $»^{30}$. Gramsci a sur ces questions un avis beaucoup plus nuancé que Raphaël Hythlodée : bien qu'il se prononce pour le "remplacement» de l'enseignement du latin, il défend la nécessité d'une forme d'apprentissage "mécanique » dans la prime enfance, par exemple par la grammaire, nécessaire selon lui pour « sélectionner de grands savants $»^{31}$. L'idée sous-jacente est que l'apprentissage aride des langues mortes doit demeurer le modèle pour la formation première des jeunes esprits, qui doivent s'accoutumer à la rigueur, se plier à un certain nombre de règles intellectuelles avant, et afin de pouvoir eux-mêmes exprimer leur propre créativité.

En effet, on trouve finalement, à l'horizon de la pédagogie gramscienne, des objectifs d'émancipation individuelle que l'on ne trouve pas développés comme tels, soulignons-le, dans la plupart des textes utopiques. Le but recherché est « l'autodiscipline intellectuelle et l'autonomie morale » qui seuls permettent l'accès, à l'issue de la formation, à « l'école de création » dans laquelle les potentialités préalablement mises en ordre, nourries de culture et informées, au plein sens du terme, donneront enfin leur pleine mesure. Le " conformisme dynamique $~^{32}$ de la première éducation, comme dit Gramsci, est ainsi à ses yeux le seul véritable moyen de conquérir la liberté. Autrement dit, on ne devient pas créatif sans une maitrise préalable des règles de l'art. Ce dernier point permet de rendre raison du paradoxe apparent de ce débat à front renversé entre des penseurs humanistes (notamment More et Campanella) qui semblent rejeter un certain héritage de l'humanisme, à savoir le dialogue avec les Anciens rendu possible par la maîtrise des langues de l'Antiquité, et un penseur socialiste qui a l'air de défendre l'école « conservatrice ». C'est l'approfondissement de la réflexion sur l'émancipation qui permet manifestement à Gramsci d'introduire une dialectique de la liberté et de la contrainte qui n'est que rarement thématisée dans l'utopie. Ni unilatéralement contraignante, ni libertaire, la pédagogie gramscienne se présente comme un processus qui passe par la contrainte pour produire la liberté. Un processus idéal : réaliste ou irréaliste ? Possible ou impraticable ? C'est là une autre question.

Le prisme de l'éducation permet peut-être de nuancer l'image souvent caricaturale que l'on se fait du rapport entre utopie et socialisme. Non pas tant parce que l'on pourrait mettre au jour un "héritage », toujours douteux, que les socialistes modernes auraient puisé dans d'hypothétiques lectures des utopistes ; mais plutôt parce qu'il est intéressant d'analyser dans quelle mesure des causes analogues produisent des effets similaires; en l'occurrence, comment un désir de changement social radical, de rationalisation du réel et d'égalisation des conditions, comment l'espérance en un monde plus moral et plus fraternel, mais également moins contraignant, amènent à poser les problèmes dans des termes proches. On espère avoir montré que le rapprochement avec les utopies permet de porter un nouvel éclairage sur le texte de Gramsci . "L'utopisme » à l'œuvre dans la pensée pédagogique de Gramsci vaut peut-être comme symptôme du fait que les pensées 
de la transformation sociale ne peuvent s'affranchir absolument d'une certaine dose d'utopie: une façon de dire que celle-ci est sans doute encore loin d'avoir épuisé son potentiel créatif et émancipateur.

\section{BIBLIOGRAPHIE}

Abensour M., 1966, « La philosophie politique de Saint-Just », Annales historiques de la Révolution française, 38e Année, pp. 1-32 (première partie) et 341-358 (deuxième partie).

Abensour Miguel, 2009, L'utopie de Thomas More à Walter Benjamin, Paris, Sens et Tonka.

Allaire, Martine et Franck Marie-Thérèse (éd.), 1995, Les politiques de l'éducation en France, Paris, La documentation française.

Baczko Bronislaw, 2000, Une éducation pour la démocratie, textes et projets de l'époque révolutionnaire, Paris, Droz.

Borg Carmel, Philipp A. Buttigieg et Peter Mayo (dir.), 2002, Gramsci and Education, Oxford, Rowman \& Littlefield.

Cabet Etienne, 1979, Voyage en Icarie, Paris/Genève, Slatkine.

Campanella Tomaso, 1972, La Cité du Soleil (1602), Genève, Droz.

Drouin-Hans Anne-Marie, 2004, Education et utopies, Paris, Vrin.

Frandji Daniel, 2015/1, « Rapport pédagogique et école unitaire dans la conceptualisation gramscienne du pouvoir », Actuel Marx, n57, p. 43-61.

Frétigné Jean-Yves, 2007, Les conceptions éducatives de Giovanni Gentile, entre élitisme et fascisme, Paris, L'Harmattan.

Gramsci Antonio, 1978-1996, Cahiers de prison (1926-1934), Paris, Gallimard.

Gramsci Antonio, 1971, Lettres de prison (1926-1934), Paris, Gallimard.

Hincker François, 1987, « L'effet d'utopie de la Révolution française » dans Matériaux pour l'histoire de notre temps, vol. $9, \mathrm{n}^{\circ} 1$, p. 2-7.

Hudde Hinde et Kuon Peter (dir.), 1988, De l'utopie à l'uchronie, Tübingen, Narr Verlag.

Keucheyan Razmig (dir.), 2012, Guerre de mouvement et guerre de position, anthologie de textes d'Antonio Gramsci, Paris, La fabrique.

Lombardi Franco, 1971, La pédagogie marxiste d'Antonio Gramsci, Toulouse, Privat.

Macherey Pierre, 2011, De l'utopie !, Le Havre, De l'incidence éditeur.

More Thomas, 1993, L'Utopie (1516), Paris, Flammarion.

Morelly Etienne-Gabriel, 2011, Le Code de la Nature (1755), Montreuil, La ville brûle.

Saint-Just, Antoine, 2004, Euvres complètes, Paris, Folio. 


\section{NOTES}

1. Voir notamment sur cette question les actes d'un colloque récent: C. Borg, P. A. Buttigieg et P. Mayo (dir.), Gramsci and Education, Oxford, Rowman \& Littlefield, 2002.

2. Antonio Gramsci, Cahiers de prison, Robert Paris (éd.), Paris, Gallimard, 1978-1996, t. III, Cahier 10 , p. 360. Nous soulignons. Désormais les citations issues des cahiers seront désignées par un $\mathrm{C}$ suivi du numéro du cahier et du numéro de la page.

3. Sur cette réforme et son principal promoteur: Jean-Yves Frétigné, Les conceptions éducatives de Giovanni Gentile, entre élitisme et fascisme, Paris, L'Harmattan, 2007.

4. C 12, p. 327. Nous soulignons.

5. M. Abensour, "La philosophie politique de Saint-Just ", Annales historiques de la Révolution française, 1966, 38è année, deuxième partie, p. 357. Voir également l'édition par le même M. Abensour des Euvres complètes de Saint-Just, Paris, Gallimard, 2004.

6. C 12, p. 327.

7. Ibid. .

8. C 12 , p. 330.

9. «Introduction » au Plan Langevin-Wallon, cité dans M. Allaire et M.-T. Franck (éd.), Les politiques de l'éducation en France, Paris, La documentation française, 1995, p. 146.

10. «Principes généraux » du Plan Langevin-Wallon dans Les politiques de l'éducation en France, op. cit., p. 147.

11. Article 4 de la loi du 11 juillet 1975, citée dans Les politiques de l'éducation en France, op. cit., p. 219.

12. C 12, p. 330

13. Ibid.

14. Voir sur ce point Daniel Frandji, «Rapport pédagogique et école unitaire dans la conceptualisation gramscienne du pouvoir », Actuel Marx, 2015/1, n57, p. 43-61.

15. C 12, p. 331.

16. Cité dans Bronislaw Baczko, Une éducation pour la démocratie, textes et projets de l'époque révolutionnaire, Paris, Droz, 2000, p. 348.

17. Cité dans Bronislaw Baczko, op. cit., p. 370. Nous soulignons.

18. Cité dans B. Baczko, op. cit., p. 349.

19. C 12, p. 330.

20. F. Hincker, «L'effet d'utopie de la Révolution française » dans Matériaux pour l'histoire de notre temps, 1987, vol. 9, $\mathrm{n}^{\circ}$ 1, p. 2-7.

21. C 12, p. 327.

22. Etienne Cabet, Voyage en Icarie, Paris/Genève, Slatkine, 1979, p. 74. Souligné par l'auteur.

23. T. More, L'Utopie, Paris, Flammarion, 1993, p. 222.

24. C12, p. 334.

25. Cité dans Anne-Marie Drouin-Hans, Education et utopies, Paris, Vrin, 2004, p. 109.

26. Gramsci, Lettre à Julia, $1^{\mathrm{er}}$ août 1932, op. cit., pp. 443-444. Nous soulignons. 
27. A.-M. Drouin-Hans, Education et utopies, op. cit.,, p. 120.

28. C 12 , p. 344.

29. C 12 , p. 339.

30. A.-M. Drouin-Hans, op. cit., p. 113.

31. C 12 , p. 339.

32. C 12 , p. 333.

\section{RÉSUMÉS}

Le présent article propose une relecture du Cahier 12 de Gramsci, consacré aux questions pédagogiques, en l'envisageant dans sa dimension utopique largement sous-estimée. En rapprochant ce texte de l'utopie canonique de Thomas More, mais également d'autres textes utopiques, il interroge le rapport complexe entre contrainte et liberté qui est au cœur de toute pédagogie visant la transformation sociale, c'est-à-dire à la fois individuelle et collective.

This paper focuses on the 12th Gramsci's Prison Notebooks, which is devoted to pedagogical matters, so as to bring its underestimated utopian dimension into light. By comparing Gramsci's claims with the canonical utopia of Thomas More as well as with other utopias, the paper investigates the complex relationship between authority and emancipation which lies at the core of any educational effort aiming for social transformation (individual and collective).

El presente artículo propone una relectura del Cuaderno 12 de Gramsci, consagrado a la temática de la pedagogía, con la intención de exponer su dimensión utópica muchas veces subestimada. Aproximando este texto a la utopía canónica de Tomás Moro, así como a otros escritos utópicos, se interroga aquí la compleja relación entre obligación y libertad que se halla en el corazón de toda pedagogía con pretensión de transformación social, vale decir a la vez individual y colectiva.

\section{INDEX}

Keywords : Gramsci, Utopia, Pedagogy, Socialism

Palabras claves : Gramsci, utopía, pedagogía, socialismo

Mots-clés : Gramsci, utopie, pédagogie, socialisme

\section{AUTEUR}

\section{STÉPHANIE ROZA}

Chercheuse au CNRS rattachée au Laboratoire IRCL de l'Université de Montpellier 3 